K. Ueno

Nagoya Math. J.

Vol. 124 (1991), 55-59

\title{
LATTICE PATH PROOF OF THE RIBBON DETERMINANT FORMULA FOR SCHUR FUNCTIONS
}

\author{
KAZUO UENO
}

In this note we give a lattice path proof of the ribbon determinant formula for Schur functions ((1) below) which was originally formulated and proved in [2].

We make use of the terminology and notation of [2]. In particular, we use the French notation of partitions and diagrams, and identify a partition with its diagram. The ribbon determinant formula for a Schur function reads:

$$
S_{J}=\operatorname{det}\left(S_{\theta_{i}^{+} \& \theta_{j}^{-}}\right)_{1 \leq i, j \leq p},
$$

where $J$ is a partition, $\left(\Theta_{p}, \ldots, \Theta_{1}\right)$ is the ribbon decomposition of $J$ with $\Theta_{i}^{+}$resp. $\Theta_{i}^{-}$the upper resp. lower part of $\Theta_{i}$, and $S_{J}$ is the Schur function for $J$.

ExAmple 1. A ribbon decomposition with $p=3$.

$$
\begin{aligned}
& J=\begin{array}{lllll}
\# & \# & \# & \\
\$ & \$ & \& & & \\
\% & \& & \# & \# & \# \\
\& & \$ & \$ & \$ & \#
\end{array} \\
& \&=\text { diagonal box },
\end{aligned}
$$

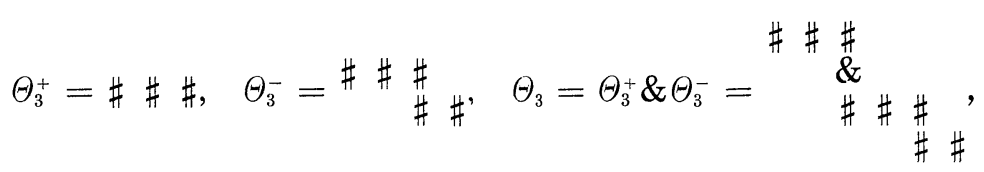

$$
\begin{aligned}
& \Theta_{2}^{+}=\$ \$, \quad \Theta_{2}^{-}=\$ \$ \$, \quad \Theta_{2}=\Theta_{2}^{+} \& \Theta_{2}^{-}=\begin{array}{l}
\$ \underset{\$}{\$} \\
\$ \$ \$
\end{array}, \\
& \Theta_{1}^{+}=\%, \quad \Theta_{1}^{-}=\text {empty, } \quad \Theta_{1}=\Theta_{1}^{+} \& \Theta_{1}^{-}=\% \text {. }
\end{aligned}
$$

Take the outermost ribbon $\Theta_{p}$. We start from the leftmost and top-

Received September 20, 1990. 
most box. Assign letter $a$ to the first box. To a box other than the first one, if the box is on the right of the preceding one, then assign letter $a$; if the box is below the preceding one, then assign letter $b$. We thus obtain a sequence of letters $a$ and $b$, which we call the assigning sequence for $J$.

ExAmple 2. To the ribbon $\Theta_{3}$ of Example 1 corresponds the assigning sequence

$$
\begin{array}{llllllllll}
a & a & a & b & b & a & a & b & a
\end{array}
$$

Note that an outermost ribbon determines a partition $J$ uniquely. For example, the ribbon $\Theta_{3}$ of Example 1 gives the partition (2) and its assigning diagram defined as

$$
\begin{array}{ccccccccc}
a & a & a & b & b & a & a & b & a \\
a & a & b & b & a & a & & \\
& a & b & & & & &
\end{array}
$$

in which the second resp. third row corresponds to the second resp. third outer ribbon. In a partition, the boxes on a particular line parallel to the diagonal assign the same letter; for instance, the diagonal boxes of (2) all assign letter $b$, and the boxes just above the diagonal all assign letter $a$. In the assigning diagram, the letters corresponding to the boxes on a particular line parallel to the diagonal are defined to be placed in the same column so that in a particular column we have all $a$ 's or all $b$ 's. We see that giving an outermost ribbon completely determines a partition and its assigning diagram.

We work with lattice paths in $\mathbb{Z} \times \mathbb{N}$ taking up-vertical, downvertical, horizontal, and south-east steps which are as vectors $(0,1)$, $(0,-1),(1,0)$ and $(1,-1)$ respectively. An up- or down-vertical step has weight 1 , and both a horizontal step of height $k$ and a south-east step at height $k$ have weight $u_{k}$, which is an indeterminate.

Let $\theta_{i}^{+}$resp. $\theta_{i}^{-}$be the number of boxes in $\Theta_{i}^{+}$resp. $\Theta_{i}^{-}$. We take as starting points $\alpha_{i}:=\left(-\theta_{i}^{+}-1,1\right)(i=1, \cdots, p)$ and as ending points $\beta_{i}:=\left(\theta_{i}^{-}, 1\right)(i=1, \cdots, p)$. We consider the lattice paths whose steps are subject to the following conditions:

(i) Let $c_{j}$ be the $j$ th letter of the assigning sequence for $J$. If $c_{j}=a$ resp. $b$, then a step starting on the line $x=-\theta_{p}^{+}-2+j$ and ending on the line $x=-\theta_{p}^{+}-1+j$ ( $x$ being the first coordinate) must 
be horizontal resp. south-east. (cf. definition of assigning diagram)

(ii) A down- resp. up-vertical step must not preceed a horizontal resp. south-east step.

We call the lattice paths under these conditions simply paths.

Let $P_{\pi}$ be the set of all $p$-tuples of paths $s=\left(s_{1}, \cdots, s_{p}\right)$ with $s_{i}$ a path from $\alpha_{i}$ to $\beta_{\pi(i)}$, where $\pi$ is a permutation of $\{1,2, \cdots, p\}$, and let $P:=\bigcup_{\pi \in G} P_{\pi}$, where $G$ is the symmetric group on $\{1,2, \cdots, p\}$.

We first show that

$$
\operatorname{det}\left(S_{\theta_{i}^{+} \& \theta_{j}^{-}}\right)_{1 \leq i, j \leq p}=\sum_{s \in P} \mathrm{Wt}(s),
$$

where $\operatorname{wt}(s)=\operatorname{sgn}(\pi) \operatorname{wt}\left(s_{1}\right) \cdots \operatorname{wt}\left(s_{p}\right)$ with $s=\left(s_{1}, \cdots, s_{p}\right) \in P_{\pi}$, and $\operatorname{wt}\left(s_{i}\right)$ is the product of the weights of all the steps appearing in $s_{i}$.

Proof of (3). The left-hand side of (3) is equal to

$$
\sum_{\pi \in G} \operatorname{sgn}(\pi) S_{\theta_{1}^{+} \& \bar{\pi}_{\bar{\pi}(1)}} \cdots S_{\theta_{p}^{+} \& \theta_{\bar{\pi}(p)}} .
$$

It suffices to show that

$$
S_{\theta_{i}^{+} \& \theta_{\bar{\pi}(i)}^{-}}=\sum_{s_{i} \in P_{\pi(i)}} \operatorname{wt}\left(s_{i}\right) \quad(i=1, \cdots, p)
$$

where $P_{\pi(i)}$ is the set of all paths from $\alpha_{i}$ to $\beta_{\pi(i)}$. Let $T_{i}$ be the set of all column-strict tableaux with shape $\Theta_{i}^{+} \& \Theta_{\pi(i)}^{-}$. Then the left-hand side of (4) is equal to $\sum_{t \in T_{i}} \mathrm{WT}(t)$, where WT is the usual indeterminate weighting for tableaux $[3,4]$, so that we have only to give a weightpreserving bijection between $P_{\pi(i)}$ and $T_{i}$. Let $s_{i} \in P_{\pi(i)}$. Read the 2nd coordinates of the ending points of all the horizontal and south-east steps appearing in $s_{i}$ in order from left to right. The number of such 2nd coordinates is $\theta_{\pi(i)}^{-}+\theta_{i}^{+}+1$, which is equal to the number of boxes in $\Theta_{i}^{+} \& \Theta_{\pi(i)}^{-}$. Write down these 2nd coordinates one by one in the boxes in order from the leftmost and topmost. The condition (i) corresponds to the condition that in a particular column of the assigning diagram for $J$ we have all $a$ 's or all $b$ 's, and the latter describes the ribbon decomposition of $J$. The condition (ii) corresponds to the condition that the array of integers on $\Theta_{i}^{+} \& \Theta_{\pi(i)}^{-}$gives a column-strict tableaux with shape $\Theta_{i}^{+} \& \Theta_{\pi(i)}^{-}$. Hence the integer sequence read off from $s_{i}$ fits into $\Theta_{i}^{+} \& \Theta_{\pi(i)}^{-}$ and yields a tableau $t \in T_{i}$.

Conversely, let $t \in T_{i}$. Read the integers in the boxes in order from the leftmost and topmost. If the first box carries integer $k$, then we draw a horizontal step from $\left(-\theta_{i}^{+}-1, k\right)$ to $\left(-\theta_{i}^{+}, k\right)$. For $j=2, \cdots, \theta_{\pi(i)}^{-}+\theta_{i}^{+}$ 
+1 , if the $j$ th box is on the right of the preceding one and carries integer $k$, then we draw a horizontal step from $\left(-\theta_{i}^{+}-2+j, k\right)$ to $\left(-\theta_{i}^{+}\right.$ $-1+j, k)$, or if the $j$ th box is under the preceding one and carries integer $k$, then we draw a south-east step from $\left(-\theta_{i}^{+}-2+j, k+1\right)$ to $\left(-\theta_{i}^{+}-1+j, k\right)$. Adding the necessary down- or up-vertical steps, we obtain a path $s_{i} \in P_{\pi(i)}$; the condition (i) is automatically satisfied and the condition (ii) corresponds to the assumption that $t$ is a ribbon columnstrict tableau. (See the last part of the reverse implication.)

We next show that

$$
S_{J}=\sum_{s \in \mathrm{NP}} \mathrm{wt}(s),
$$

where NP denotes the set of all nonintersecting $p$-tuples of paths $s=$ $\left(s_{1}, \cdots, s_{p}\right)$ with $s_{i}$ a path from $\alpha_{i}$ to $\beta_{i}(i=1, \cdots, p)$.

Proof of (5). Let $T$ be the set of all column-strict tableau with shape $J$. Then the left-hand side of (5) is equal to $\sum_{t \in T} \mathrm{WT}(t)$ (see the proof of (3)), so that we have only to construct a weight-preserving bijection between NP and $T$. Let $s=\left(s_{1}, \cdots, s_{p}\right) \in$ NP. The proof of (3) with $\pi=$ id gives a ribbon column-strict tableau $t_{i}$ with shape $\Theta_{i}=\Theta_{i}^{+} \& \Theta_{i}^{-}$corresponding to $s_{i}(i=1, \cdots, p)$. We compose an array $t$ of integers with shape $J$ from $t_{i}(i=1, \cdots, p)$ according to the ribbon decomposition $\left(\Theta_{p}\right.$, $\cdots, \Theta_{1}$ ) of $J$. Since $s$ is nonintersecting, $t$ is in fact a column-strict tableau, i.e. $t \in T$. (See Example 3 below.)

Conversely, let $t \in T$. We can reverse the above procedure to obtain $s \in \mathrm{NP}$ corresponding to the tableau $t$.

Example 3. To the tableau

$$
\begin{array}{lllll}
5 & 5 & 5 & & \\
3 & 4 & 4 & & \\
2 & 2 & 2 & 3 & 4 \\
1 & 1 & 1 & 2 & 2
\end{array}
$$

with shape (2) corresponds the nonintersecting 3-tuple of paths:

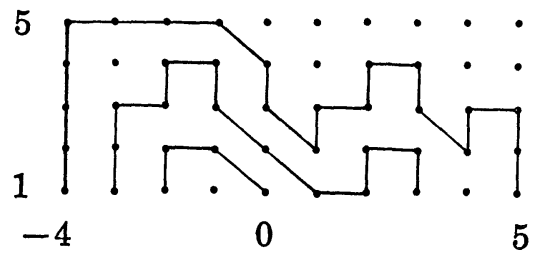


Finally we give:

Proof of (1). In view of (3) and (5), it suffices to show that (6)

$$
\sum_{s \in P} \mathrm{wt}(s)=\sum_{s \in \mathrm{NP}} \mathrm{wt}(s),
$$

which we see using the Gessel-Viennot method [1,5]; in fact we can apply [1, Corollary 2] or [5, Theorem 1.2] to obtain (6) by noting that, if $s \in P_{\pi}$ is nonintersecting, then $\pi$ must be the identity permutation.

\section{REFERENCES}

[1] I. M. Gessel and G. Viennot, Determinants, paths, and plane partitions, preprint, July 1989.

[2] A. Lascoux and P. Pragacz, Ribbon Schur functions, Europ. J. Combinatorics, 9 (1988), 561-574.

[ 3 ] I. G. Macdonald, Symmetric Functions and Hall Polynomials, Oxford Univ. Press, 1979.

[ 4 ] D. Stanton and D. White, Constructive Combinatorics, Springer, New York, 1986.

[ 5 ] J. R. Stembridge, Nonintersecting paths, Pfaffians, and plane partitions, Advances in Math., 83 (1990), 96-131.

Department of Mathematics

Nagoya Institute of Technology

Showa-ku, Nagoya 466

Japan 
